\title{
VALIDAÇÃO DE CONTEÚDO: APLICATIVO MÓVEL SAFE HEART PARA MONITORAMENTO E IDENTIFICAÇÃO DE RISCO DE INFARTO
}

\author{
CONTENT VALIDATION: SAFE HEART MOBILE \\ APPLICATION FOR MONITORING AND \\ IDENTIFICATION OF INFARCTION RISK
}

\section{VALIDACIÓN DE CONTENIDO: APLICACIÓN MÓVIL SAFE HEART PARA EL SEGUIMIENTO E IDENTIFICACIÓN DEL RIESGO DE INFARTO}

\author{
Minéia Rossette de Souza ${ }^{1}$ \\ Maria Luiza Carvalho de Oliveira ${ }^{2}$ \\ Eveline Menezes Caçote Barbosa ${ }^{3}$ \\ Elielza Guerreiro Menezes ${ }^{4}$ \\ Gladson Souza de Araújo5 \\ Suelem Maciel Nascimento ${ }^{6}$ \\ Daniely Bianca Magalhães de Figueiredo Carvalho ${ }^{7}$
}

\begin{abstract}
Como citar este artigo: Souza MR, Oliveira MLC, Barbosa EMC, Menezes EG, Araújo GS, Nascimento SM, et al. Validação de conteúdo: aplicativo móvel Safe Heart para monitoramento e identificação de risco de infarto. Rev baiana de enferm. 2021;35:e44403.

Objetivo: verificar a validade de conteúdo do aplicativo móvel Safe Heart para monitoramento e identificação de risco de infarto. Método: estudo de validação de conteúdo. Participaram do estudo 10 juízes. Foram avaliados 21 itens, por meio da escala de Likert. Para estimativa do grau de concordância, foi utilizado o cálculo do coeficiente alfa de Cronbach. Resultados: a análise estatística com alpha de Cronbach 0,9573 consolidou o Safe Heart quanto à validade e à confiabilidade na consistência interna do conteúdo desenvolvido no aplicativo; os juízes apresentaram consistência válida nos itens observados. Conclusão: a validade de conteúdo do aplicativo móvel Safe Heart foi aprovada para monitoramento e identificação de risco de infarto.
\end{abstract}

Descritores: Tecnologia da Informação. Estudos de Validação. Tecnologia em Saúde.

Objective: to verify the content validity of the Safe Heart mobile app for monitoring and identification of infarction risk. Method: content validation study. Ten judges participated in the study. Twenty-one items were evaluated using the Likert scale. To estimate the degree of agreement, Cronbach's alpha coefficient was calculated. Results: Cronbach's alpha statistical analysis with 0.9573 consolidated Safe Heart regarding validity and reliability in the internal

\footnotetext{
Enfermeira. Teóloga. Especialista em Enfermagem do Trabalho. Universidade do Estado do Amazonas. Manaus, Amazonas, Brasil. https://orcid.org/0000-00030430-3722.

Enfermeira. Doutora em Enfermagem. Docente da Universidade do Estado do Amazonas. Manaus, Amazonas, Brasil. mlcoliveira@uea.edu.br. https://orcid.org/0000-0002-1077-1066.

Enfermeira. Mestre em Enfermagem. Docente da Universidade do Estado do Amazonas. Manaus, Amazonas, Brasil. https://orcid.org/0000-0002-3009-9/82.

Enfermeira. Doutora em Enfermagem. Docente da Universidade do Estado do Amazonas. Manaus, Amazonas, Brasil. https://orcid.org/0000-0003-1804-6384

Estudante de Engenharia de Computação. Universidade do Estado do Amazonas. Manaus, Amazonas, Brasil. https://orcid.org/0000-000 I-6221-7799.

Estudante de Engenharia da Computação. Universidade do Estado do Amazonas. Manaus, Amazonas, Brasil. https://orcid.org/0000-0002-3340-0954

Estudante de Enfermagem. Universidade do Estado do Amazonas. Manaus, Amazonas, Brasil. https://orcid.org/0000-0003-096 I-5872.
} 
consistency of the content developed in the application; the judges presented valid consistency in the observed items. Conclusion: the content validity of the Safe Heart mobile app has been approved for monitoring and identification of infarction risk.

Descriptors: Information Technology. Validation Studies. Health Technology.

Objetivo: verificar la validez de contenido de la aplicación móvil Safe Heart para el seguimiento e identificación del riesgo de infarto. Método: estudio de validación de contenido. Diez jueces participaron en el estudio. Veintiún items fueron evaluados mediante la escala Likert. Para estimar el grado de concordancia, se calculó el coeficiente alfa de Cronbach. Resultados: el análisis estadístico con Cronbach de alfa 0,9573 consolidó Safe Heart en cuanto a validez y confiabilidad en la consistencia interna del contenido desarrollado en la aplicación; los jueces presentaron consistencia válida en los ítems observados. Conclusión: la validez de contenido de la aplicación móvil Safe Heart ha sido aprobada para el seguimiento e identificación del riesgo de infarto.

Descriptores: Tecnologías de la Información. Estudios de Validación. Tecnología en Salud.

\section{Introdução}

O termo "tecnologia" é formado pelos radicais gregos tekhne, que significa "técnica, arte, ofício", e logos, que se refere a "conjunto dos saberes". A tecnologia é um objeto de estudo constante da ciência e da engenharia. Com o avanço tecnológico, as pessoas têm a possibilidade de contato com sistemas computacionais, o que permite a melhoria de processos em diversas áreas do conhecimento e novas possibilidades e desafios para interações entre pessoas e máquinas ${ }^{(1-2)}$.

Dentre a diversidade de tecnologias, destaca-se a preditiva, que combina uma série de técnicas e fórmulas matemáticas e estatísticas, como machine learning e data mining, para coletar dados em tempo real e, em seguida, apontar tendências, identificar riscos e fazer prognósticos ${ }^{(3)}$.

$\mathrm{Na}$ área da saúde, modelos preditivos permitem a identificação de padrões existentes, incluindo variáveis demográficas e comportamentais dos pacientes. Esses modelos resultantes identificam, além de segmentos de uma população de pacientes possíveis candidatos de alto custo, os fatores que levam os pacientes a terem o perfil de custo elevado. Por meio de modelos de análise preditiva, ainda é possível realizar previsões de taxas de internação, probabilidade de infecções e doenças, assim como predizer potenciais pacientes para programas de saúde e bem-estar. Seus resultados, quando combinados a medidas de saúde pública aplicadas no plano populacional, podem trazer implicações positivas na redução de custos e na efetividade de intervenções, como tratamentos e ações preventivas $^{(3-4)}$.

Atrelado a esse avanço tecnológico na saúde, percebe-se o crescente uso da tecnologia móvel na sociedade, influenciando na busca de informação e na interatividade com os usuários. Os dispositivos móveis, como smartphones, tablets, notebook têm sido utilizados em diversos contextos para dinamizar o fluxo de dados de informação, contribuindo para a produção de conhecimento em redes e a ampliação da comunicação em serviços de saúde ${ }^{(2)}$.

A popularização de smartphones, os celulares inteligentes, tem sido considerada uma revolução tecnológica de maior impacto, com alto crescimento de dispositivos no mercado. Com isso, os aplicativos para telefone móvel fazem cada vez mais parte da rotina das pessoas, pois podem ser usados para diversas finalidades, como: chamar um carro particular, pedir uma refeição, baixar músicas e assistir a vídeos. Mais de 300 bilhões de aplicativos são baixados por ano pela proatividade, diversidade e facilidade de acesso ${ }^{(5)}$.

Aplicativos móveis para a saúde têm como objetivo principal auxiliar o cuidado com a saúde dos usuários. Com a finalidade de abranger conceitos referentes à utilização de meios tecnológicos aplicados nos atendimentos de cuidados em saúde, a Organização Mundial em Saúde 
(OMS) definiu os termos eHelthe mHealth. Sendo assim, os aplicativos móveis tornaram-se importantes ferramentas de suporte no acolhimento ao paciente, na medida em que viabilizam os procedimentos do cuidado, além de disponibilizarem o acesso à informação de maneira célere e segura, solucionando problemas e otimizando atividades realizadas no dia a dia ${ }^{(6)}$.

Atreladas aos aplicativos móveis, as tecnologias consideradas vestíveis também têm se destacado no mercado tecnológico, como os smartwatches, em português chamados de relógios inteligentes. Os smartwaches são muito utilizados para auxiliar nos cuidados de monitorização, verificação e notificação para o usuário. Quando integrados com aplicativos móveis, a funcionalidade do sistema amplia-se, permitindo maior confiabilidade e auxílio na saúde do usuário ${ }^{(7-8)}$.

Nesse sentido, uma equipe de enfermeiros idealizou, projetou e desenvolveu um aplicativo móvel para monitoramento e identificação de risco de infarto. Trata-se de um aplicativo móvel integrado ao smartwatch denominado $S A F E$ HEART desenvolvido pela equipe do Laboratório de Tecnologia em Saúde e Educação (LABTECS) da Universidade do Estado do Amazonas (UEA) para o sistema Android, financiado pela Lei no $8.387 / 1991^{(9)}$. O LABTECS, grupo de pesquisa credenciado pelo diretório de pesquisa da Coordenação de Aperfeiçoamento de Pessoal de Nível Superior (CAPES), é composto por uma equipe de enfermeiros doutores e pesquisadores da área de tecnologia em saúde. A infraestrutura do LABTECS é constituída de uma área de 106,60 $\mathrm{m}^{2}$, dividida em cinco ambientes destinados à execução dos projetos voltados para saúde e tecnologia.

O produto foi desenvolvido no ano de 2019 por uma equipe de quatro enfermeiros, um tecnólogo em informática, um design, três desenvolvedores de aplicativos móveis e dois testers. Para utilizá-lo, é necessário que o usuário baixe o aplicativo pelo Play Store, instale o aplicativo no seu smartphone de sistema Android e sincronize com o smartwatch.

O SAFE HEARTé integrado ao smartwatch que monitora os batimentos cardíacos, analisando frequência e ritmo. Auxilia o indivíduo a identificar as alterações referentes à frequência e ao ritmo cardíacos, fatores relacionados a doenças cardiovasculares que podem comprometer a vida do usuário. A integração com o smartwatch é necessária, para que parâmetros cardíacos sejam avaliados frequentemente, enquanto o indivíduo estiver fazendo uso. Desse modo, quando for identificada uma alteração no padrão do ritmo e da frequência cardíacas, é emitido um alerta, a fim de que o usuário possa evitar complicações na sua saúde.

O aplicativo SAFE HEART analisa os parâmetros durante o sono, repouso e atividades físicas, para identificar o padrão do usuário. Seu uso pode evitar a morte por doenças cardiovasculares, pois emite alerta em tempo hábil, para que se procure atendimento médico. Tem ainda algumas funcionalidades, tais como: identificar os fatores de risco do usuário, por meio da leitura das respostas de um questionário de risco; estabelecer o grau de risco de infarto, se baixo, médio ou alto; registrar uma variância da frequência cardíaca, emitir alertas de alterações e função de geolocalização, caso identifique alto risco de infarto no usuário.

Dessa forma, o aplicativo SAFE HEART impacta diretamente no usuário e na família, ao emitir alertas que antecipam eventos adversos que podem culminar com a morte. Ao receber a notificação de alerta, o usuário poderá antecipar e providenciar socorro imediato, acionando o sistema de saúde local.

O objetivo deste artigo é verificar a validade de conteúdo do aplicativo móvel SAFE HEART integrado ao samrtwatch para monitoramento e identificação de risco de infarto.

\section{Método}

Pesquisa de validação de conteúdo do aplicativo móvel SAFE HEART integrado ao smartwatch para identificação de risco de infarto agudo do miocárdio. O processo de validação consiste em um julgamento útil para a tomada de decisões, fornecendo ao pesquisador garantia das escolhas efetivas para a produção e 
o sucesso de um produto. Tem por objetivo a avaliação da qualidade de um produto e de seus componentes e é realizada por especialistas da área que se quer avaliar ${ }^{(10)}$.

Participaram como juízes deste estudo dez enfermeiros especialistas de uma instituição pública de Manaus (AM). A seleção da amostra composta por conveniência foi realizada pelo modelo conhecido como snowball ou bola de neve, uma forma de seleção não probabilística.

Não há um número pré-estabelecido para a escolha dos juízes para se validar os resultados obtidos. Esse número pode variar de acordo com o fenômeno estudado e com os critérios para a seleção. Sendo assim, o critério de inclusão, no caso desta pesquisa, seguindo a recomendação de estudos similares ${ }^{(11-12)}$, foi o enfermeiro atingir o mínimo de três e o máximo de dez pontos, de acordo com as seguintes condições: doutorado em enfermagem (4 pontos); docente com mestrado em educação ou em enfermagem (3 pontos) e desenvolvimento de tecnologias em saúde ou educação (2 pontos); docente em enfermagem (1 ponto).

Foi realizada a busca ativa de enfermeiros com expertise em saúde e tecnologia na Plataforma Lattes. Assim, foram convidados dez juízes, por meio da carta convite digital. Após o aceite, foi enviado o Termo de Consentimento Livre Esclarecido (TCLE), conforme determina a Resolução n. 466/2012 (13) , que, após assinado, confirmou a participação no estudo.

A coleta de dados, diante dos riscos de transmissão do novo coronavírus, ocorreu nas dependências do LABTECS, no mês de março de 2021, seguindo todos as recomendações contidas no plano de cuidados sanitários na condução de pesquisa. Os juízes utilizaram o aplicativo móvel integrado ao smartwatch em ambiente de teste, com o aplicativo instalado em um smartphone Samsung Galaxy S1O e um smartwatch Samsung Galaxy Watch 2. O tempo das avaliações teve duração média de 30 minutos.

Os instrumentos de coleta de dados foram um aplicativo móvel SAFE HEART integrado ao smartwacth e dois formulários: um de dados sociodemográficos, para caracterização dos juízes; e outro de avaliação do conteúdo do aplicativo móvel SAFE HEART. Este último foi construído por meio da adaptação de um questionário validado, fundamentado na ISO/IEC 12207 e ISO/DIS 9241-11 ${ }^{(14)}$. Nas respostas, foi utilizada a escala de Likert de 1 a 4 pontos, que considerou os seguintes escores: (1) irrelevante; (2) pouco relevante; (3) relevante; (4) muito relevante.

A análise dos dados referentes à caracterização e avaliação dos juízes foi organizada em uma planilha eletrônica do Programa Microsoft Excel. Os dados foram apresentados na forma de frequências relativas e absolutas.

Para a validação de conteúdo, cada item do instrumento de avaliação foi pontuado numa escala de Likert. Na sequência, para estimativa do grau de concordância entre os juízes e para fornecer evidências da precisão e confiabilidade do teste de pontuações, foi utilizado o cálculo do coeficiente alfa de Cronbach na observação de concordância interna para validação. Dessa forma, quanto menor a variância, mais consistente é o conteúdo avaliado. Se o valor do coeficiente alfa de Cronbach for baixo, a correlação média entre os itens avaliados também será. Portanto, o valor mínimo aceitável para o alfa é 0,70; abaixo desse valor, a consistência interna da escala utilizada é considerada baixa ${ }^{(15-16)}$.

Quanto às considerações éticas da pesquisa, este estudo faz parte do macroprojeto intitulado "Desenvolvimento e Validação de Tecnologias Interativas em Saúde e Educação na Inovação do Cuidado Humano" do LABTECS. Seguindo a Resolução n. 466/2012 ${ }^{(13)}$, do Conselho Nacional de Saúde, foi enviado ao Comitê de Ética e Pesquisa da Universidade do Estado do Amazonas, que o aprovou pelo Parecer $n^{0}$ 3.574.288 e Certificado de Apresentação de Apreciação Ética (CAEE) 15924919.6.0000.5016.

\section{Resultados}

Dentre os juízes que aceitaram participar do estudo, nove eram do sexo feminino e um do sexo masculino. A média de idade foi de 41 anos, correspondendo a uma faixa etária entre 27 e 57 anos. Quanto à formação acadêmica, todos eram graduados em Enfermagem, com média de tempo de formado de 14,9 anos, sendo o mais 
experiente graduado em 2003 e o mais novato, formado em 2018. Todos os juízes possuíam pós-graduação lato sensu nas mais variadas especialidades: um em Enfermagem do Trabalho, dois em Gestão de Saúde, dois em Urgência e Emergência, um em Saúde Mental, um em Enfermagem Cirúrgica, um em Administração Hospitalar, um em Hemoterapia e um em Enfermagem em Alta Complexidade. Ainda sobre a formação, quatro juízes eram doutores, dois mestres e quatro especialistas.

No que se refere à atuação profissional dos juízes, quatro trabalhavam exclusivamente na assistência e seis na docência de nível superior.
Nove dentre eles participavam ou já teriam participado de pesquisas na área de tecnologia e saúde.

De acordo com os critérios de inclusão, os juízes deveriam atingir o mínimo de três e máximo dez pontos nos itens: para enfermeiros - doutorado em enfermagem (4 pontos), professores com mestrado em educação em enfermagem (3 pontos), desenvolvimento de tecnologias em enfermagem educação (2 pontos), professor de enfermagem (1 ponto). A Tabela 1 exibe a pontuação alcançada pelos juízes, de acordo com esse critério.

Tabela 1 - Pontuação alcançada pelos juízes especialistas. Manaus, Amazonas, Brasil - 2021. (N=10)

\begin{tabular}{l|c|c|c}
\hline Juízes Especialistas & $\mathbf{n}$ & Pontuação & $\mathbf{\%}$ \\
\hline Enfermeiros & & & 40,00 \\
Doutores & 10 & 4 & 40,00 \\
Especialistas & 6 & 4 & 20,00 \\
Mestres & 3 & 2 & 100,00 \\
Total & 10 & $\ldots$ & $\ldots$ \\
Média & $\ldots$ & 7,0 & . \\
\hline
\end{tabular}

Fonte: Elaboração própria.

Nota: Sinal convencional utilizado:

. . Não se aplica dado numérico.

Em relação à pontuação alcançada pelos juízes, obteve-se uma média de 7,0 pontos, em que o mínimo foi quatro e o máximo, sete pontos. Observa-se que 80\% dos juízes tiveram pontuação acima da média e apenas 20\% obtiveram a pontuação mínima exigida.

Quanto à validade de conteúdo do aplicativo móvel SAFE HEART, foi empregado o coeficiente de alfa de Cronbach para observação de concordância interna para validação. Foram avaliados
21 itens referentes ao aplicativo. A avaliação do grau de concordância foi calculada com base na proporção de assertivas que atingiram os escores (3) Relevante e (4) Muito Relevante.

Na Tabela 2, pode-se observar os resultados do coeficiente de alfa de Cronbach de 0,9573, considerado um dos estimadores da consistência interna. Isto significa que as questões respondidas pelos juízes apresentaram consistência válida nos itens observados.

Tabela 2 - Validação de Conteúdo do aplicativo móvel SAFE HEART. Manaus, Amazonas, Brasil - 2021

\section{Itens Avaliados}

1. O App SAFE HEART será útil no seu trabalho?

2. Eu estou satisfeita(o) em utilizar este App SAFE HEART

3. Ao conhecer a aplicação do $A p p S A F E H E A R T$, eu penso que ele ajudará a economizar o tempo para desenvolver minhas atividades

4. Eu pretendo utilizar APD SAFE HEART na minha prática profissional

5. Foi simples usar $A P p S A F E$ HEART, e eu entendi as questões por ele direcionadas

6. Eu consegui realizar minhas atividades, utilizando o App SAFE HEART

7. O App SAFE HEART não substitui minhas ações; ele me ajuda a decidir o melhor caminho para a tomada de decisão (continua)

Alfa de Cronbach 
Tabela 2 - Validação de Conteúdo do aplicativo móvel SAFE HEART. Manaus, Amazonas, Brasil - 2021

\begin{tabular}{|c|c|c|}
\hline \multicolumn{2}{|c|}{ Itens Avaliados } & \multirow{2}{*}{$\frac{\text { Alfa de Cronbach }}{0,9546}$} \\
\hline 8. & Eu me senti confortável em usar o App SAFE HEART & \\
\hline 9. & Foi fácil aprender a usar o $A P p S A F E H E A R T$ & 0,9537 \\
\hline 10. & $\begin{array}{l}\text { Eu acredito que poderia me tornar rapidamente mais produtiva(o) usando } \\
\text { APp SAFE HEART }\end{array}$ & 0,9525 \\
\hline 11. & $\begin{array}{l}\text { O App SAFE HEART fornece mensagens claras de erro, informando como } \\
\text { corrigir algum problema ou decisão equivocada }\end{array}$ & 0,9516 \\
\hline 12. & $\begin{array}{l}\text { Se eu cometo um erro, com o } A p p \text { SAFE HEART, eu posso fácil e } \\
\text { rapidamente recuperar meus dados já armazenados }\end{array}$ & 0,9512 \\
\hline 13. & $\begin{array}{l}\text { As informações fornecidas pelo App SAFE HEART (mensagens, questões, } \\
\text { opções e outros documentos) são claras }\end{array}$ & 0,9507 \\
\hline 14. & É fácil navegar no $A p p S A F E$ HEART para encontrar a informação que necessito & 0,9505 \\
\hline 15. & $\begin{array}{l}\text { As informações do App SAFE HEART estão organizadas adequadamente e } \\
\text { contemplam minha avaliação diária }\end{array}$ & 0,9504 \\
\hline 16. & $\begin{array}{l}\text { A interface do App SAFE HEART é agradável (cores, imagem, disposição } \\
\text { dos itens, navegação etc.) }\end{array}$ & 0,9504 \\
\hline 17. & Eu gostei de usar a interface do App SAFE HEART & 0,9507 \\
\hline 18. & O App SAFE HEART tem todas as funções que eu esperava & 0,9514 \\
\hline & Acima de tudo, eu estou satisfeito com o App SAFE HEART & 0,9520 \\
\hline 20. & É simples e fácil usar o $A p p$ SAFE HEART & 0,9525 \\
\hline \multirow[t]{2}{*}{21.} & $\begin{array}{l}\text { A organização e a disposição das informações nas telas do App SAFE } \\
H E A R T \text { são claras e objetivas }\end{array}$ & 0,9512 \\
\hline & Todos & 0,9573 \\
\hline
\end{tabular}

Fonte: Elaboração própria.

Os resultados demonstraram que os itens avaliados são homogêneos e medem, de forma consistente, as características do aplicativo móvel avaliado. Portanto, são confiáveis e comprovam a validade de conteúdo.

\section{Discussão}

Os aplicativos móveis ganharam força na área da saúde, com uma imensa variedade de opções de aplicativos que vão desde sistemas fitness ao monitoramento e controle de doenças. No entanto, o desenvolvimento de um aplicativo móvel para a saúde requer coerência e adequação, além do reconhecimento da necessidade do usuário. Por isso, a validação de um aplicativo desenvolvido é importante para verificar demandas específicas relacionadas à saúde ${ }^{(17)}$.

Para tanto, é essencial a formação de um comitê de juízes especialistas. Além disso, o pesquisador precisa determinar os critérios de acordo com os objetivos do estudo. No entanto, independentemente do que se deseja validar, e mesmo que o pesquisador utilize critérios próprios ou adaptados, é primordial estabelecer e respeitar os requisitos necessários para considerar um enfermeiro expert ${ }^{(18)}$.

Em estudos de validação, a seleção dos juízes especialistas é essencial para promover a acurácia do conteúdo do aplicativo, pois a escolha inadequada pode influenciar na confiabilidade dos resultados ${ }^{(11)}$. De acordo com os critérios de inclusão, nesta pesquisa, 40\% dos especialistas atingiram a pontuação máxima. Além disso, a análise do currículo lattes permitiu que fossem convidados enfermeiros com expertise do conteúdo a ser avaliado.

No Brasil, a Plataforma Lattes integra as bases de dados de currículos, grupos de pesquisa e instituições em um único sistema de informação. A busca, nessa plataforma, permite a seleção de especialistas altamente qualificados ${ }^{(19)}$. Nesta pesquisa, a amostragem de juízes do tipo snowball e a análise do currículo lattes foram etapas que garantiram a seleção favorável para o sucesso da validação. Por outro lado, o número de juízes especialistas selecionados condiz com o apontado na literatura, que recomenda o 
mínimo de cinco a seis participantes ${ }^{(19)}$. O estudo foi realizado com dez enfermeiros, e isso garantiu a diversidade de informações e robustez dos resultados.

Neste estudo, os formulários com 21 itens para avaliar o conteúdo do aplicativo móvel SAFE HEART foram enviados digitalmente. Os resultados mostraram consistência interna desejável e apresentaram homogeneidade nos itens avaliados, resultando na validade de conteúdo do aplicativo. Para que os itens avaliados apresentassem consistência interna satisfatória, deveriam possuir alpha de Cronbach superior a $0,7^{(15)}$. Neste sentido, é possível afirmar que a confiabilidade do aplicativo móvel SAFE HEART está adequado, uma vez que apresentou alpha de Cronbach 0,9573.

Desta forma, o aplicativo móvel SAFE HEART possui qualidade de conteúdo e contribui para a tomada de decisão do usuário em risco de infarto. É útil para acessar, coletar informações, monitorar a frequência cardíaca, identificar o grau de risco de infarto e emitir notificações para o usuário.

Como perspectivas futuras, pretende-se melhorar e agregar outras funcionalidades no aplicativo, para aperfeiçoar o monitoramento da saúde do usuário.

O estudo apresenta limitações no que tange ao uso aberto do aplicativo móvel SAFE HEART, pois continua em testes, com inserção de novas funcionalidades, e só pode ser utilizado em ambientes de teste específico.

Espera-se, com esta pesquisa, despertar o interesse de outros enfermeiros, para o desenvolvimento de novas tecnologias que alinhem o conhecimento teórico-prático de enfermagem, em busca da melhoria da qualidade da saúde dos usuários da tecnologia.

\section{Conclusão}

O estudo de validação do conteúdo do $S A F E$ HEART permitiu concluir-se que o aplicativo móvel foi aprovado. A análise estatística com alpha de Cronbach 0,9573 consolidou o SAFE HEART quanto à validade e à confiabilidade na consistência interna do conteúdo desenvolvido no aplicativo.

\section{Colaborações:}

1 - concepção, projeto, análise e interpretação dos dados: Maria Luiza Carvalho de Oliveira, Eveline Menezes Caçote Barbosa e Elielza Guerreiro Menezes;

2 - redação do artigo e revisão crítica relevante do conteúdo intelectual: Mineia Rossete de Souza, Gladson Souza de Araújo, Suelem Maciel do Nascimento e Daniely Bianca Magalhaes de Figueiredo Carvalho;

3 - aprovação final da versão a ser publicada: Maria Luiza Carvalho de Oliveira.

\section{Referências}

1. Barros JP, Kanda JY, Vieira AMCAP. Aplicação de técnicas de usabilidade para avaliação do aplicativo móvel Quimi Analysis. Nuevas ideas Inform Educ [Internet]. 2017 [cited 2021 Apr 11];13:551-6. Available from: http://www.tise.cl/ volumen13/TISE2017/76.pdf

2. Nicolau LASL, Rocha PC, Bandeira AMB. Uso do aplicativo móvel na promoção de saúde de pessoas com fissuras labiopalatinas: relato de experiência. Reva Acad Cient Saúde. 2019:4(1): 14-21. DOI: 10.24118/reva1806.9495.4.1.2019.480

3. Santos HG, Nascimento CF, Izbicki R, Duarte YAO, Chiavegatto Filho ADP. Machine learning para análises preditivas em saúde: exemplo de aplicação para predizer óbito em idosos de São Paulo, Brasil. Cad Saúde Pública. 2019;35(7):e00050818. DOI: 10.1590/0102-311X00050818

4. Novaes HMD, Soárez PC. A avaliação das tecnologias em Saúde: origem, desenvolvimento e desafios atuais. Panorama internacional e Brasil. Cad Saúde Pública. 2020;36(9):e:00006820. DOI: https://doi.org/10.1590/0102-311X00006820

5. Galvão AMN, Dalbon VA, Mota LMLA, Moura FRE, Magalhães AKG, Menezes ASOP, et al. A usabilidade de novas tecnologias "Up Ferida" para monitoramento de pacientes diabéticos com feridas na Atenção Básica: um estudo prospectivo. Res Soc Dev. 2021:10(1):e4201011111906. DOI: http://dx.doi. org/10.33448/rsd-v10i1.11906

6. Oliveira BL, Vilhena BJ, Freitas RN, Bastos ZRG, Teixeira E, Menezes EG, et al. Aplicativos móveis no cuidado em saúde: uma revisão integrativa. Rev Enferm Atual In Derme. 2020;93(31): 
e-020047. DOI: https://doi.org/10.31011/reaid2020-v.93-n.31-art.760

7. Silva LKGNM, Fernandes SMM, Lins RC. Uso de SmartWatch no Auxílio a Monitoração de Arritmias Cardíacas. Braz J Develop. 2020;6(10): 75511-25. DOI:10.34117/bjdv6n10-108

8. Veiga J, Rodriguez JP, Trevizan B, Rebonatto MT, Marchi ACB. Aplicações móveis com interação médico-paciente para estilo de vida saudável: uma revisão sistemática. Reciis - Rev Eletron Comun Inf Inov Saúde. 2017;11(1):1-9. DOI: https://doi.org/10.29397/reciis.v11i1.1188

9. Brasil. Ministério da Saúde. Lei n ${ }^{\circ} 8.387$, de 30 de dezembro de 1991. Dá nova redação ao $₫ 1^{\circ}$ do art. $3^{\circ}$ aos arts. $7^{\circ}$ e $9^{\circ}$ do Decreto-Lei no 288 , de 28 de fevereiro de 1967, ao caput do art. 37 do Decreto-Lei no 1.455 , de 7 de abril de 1976 e ao art. 10 da Lei no 2.145, de 29 de dezembro de 1953, e dá outras providências [Internet]. Brasília (DF); 1991 [cited 2021 Apr 11]. Available from: http:// www.planalto.gov.br/ccivil_03/leis/18387.htm

10. Penha J, Fernandes F, Oliveira C, Oliveira R, Barros E. Validação e utilização de novas tecnologias na saúde e educação: uma revisão integrativa. Rev Interdisciplin Promoç Saúde. 2018;1(3):199-206. DOI: https://doi.org/10.17058/ rips.v1i3.12580

11. Menezes EG. Validação do Processo de Enfermagem Informatizado em aplicativo móvel iNurse de acordo com a NBR ISO 25051 [Internet]. [tese]. Florianópolis: Universidade Federal de Santa Catarina; 2018. [cited 2021 Apr 14]. Available from: https://repositorio.ufsc.br/ handle/123456789/215011

12. Pereira FGF, Rocha DJL, Melo GAA, Jaques RMPL, Formiga LMF. Construção e validação de aplicativo digital para ensino de instrumentação cirúrgica. Cogitare Enferm. 2019;24:e58334. DOI: dx.doi. org/10.5380/ce.v24i0.58334

13. Brasil. Ministério da Saúde. Resolução no 466, de 12 de dezembro de 2012. Aprova diretrizes e normas regulamentadoras de pesquisas envolvendo seres humanos [Internet]. Brasília
(DF); 2012 [cited 2021 Jan 8]. Available from: http://conselho.saude.gov.br/resoluções/2012/ Reso466.pdf

14. Dal SGTM, Barra DCC, Paese F, Almeida SRW, Rios GC, Marinho MM, et al. Processo de enfermagem informatizado: metodologia para associação da avaliação clínica, diagnósticos, intervenções e resultados. Rev esc enferm USP. 2013;47(1):242-9. DOI: https://doi.org/10.1590/ S0080-62342013000100031

15. Melo EBM, Primo CC, Romero WG, Sant'Anna HG, Sequeira CAC, Lima EFA, et al. Construção e validação de aplicativo móvel para o desenvolvimento de histórico e diagnóstico de enfermagem. Rev Bras Enferm. 2020;73(Suppl 6):e20190674. DOI: https://doi.org/ 10.1590/0034-7167-2019-0674

16. Bujang MA, Omar ED, Baharum NA. A Review on Sample Size Determination for Cronbach's Alpha Test: A Simple Guide for Researchers. Malaysian J Med Sci. 2018:25(6):85-99. DOI: https://doi. org/10.21315/mjms2018.25.6.9

17. Vêscovi SJB, Primo CC, Sant'Anna HG, Bringuete MEO, Rohr RV, Prado TN, et al. Aplicativo móvel para avaliação dos pés de pessoas com diabetes mellitus. Acta paul. enferm. 2017;30(6):607-13. DOI:. https://doi. org/10.1590/1982-0194201700087

18. Catunda HLO, Bernardo EBR, Vasconcelos CTM, Moura ERF, Pinheiro AKB, Aquino PS. Percurso metodológico em pesquisas de enfermagem para construção e validação de protocolos. Texto contexto - enferm. 2017;26(2):e00650016. DOI: https://doi.org/10.1590/0104-07072017000650016

19. Vieira TW, Sakamoto VTM, Moraes LC, Blatt CR, Caregnato RCA. Validation methods of nursing protocols: an integrative review. Rev Bras Enferm. 2020;73(Suppl 5):e20200050. DOI: https://doi. org/10.1590/0034-7167-2020-0050

Recebido: 22 de abril de 2021 Aprovado: 28 de junho de 2021 Publicado: 3 de agosto de 2021

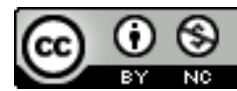

A Revista Baiana de Enfermagem utiliza a Licença Creative Commons - Atribuição-NãoComercial 4.0 Internacional. https://creativecommons.org/licenses/by-nc/4.0/ Este artigo é de acesso aberto distribuído sob os termos da Licença Creative Commons (CC BY-NC). Esta licença permite que outros remixem, adaptem e criem a partir do seu trabalho para fins não comerciais. Embora os novos trabalhos tenham de lhe atribuir o devido crédito e não possam ser usados para fins comerciais, os usuários não têm de licenciar esses trabalhos derivados sob os mesmos termos. 